\title{
Natural inflation with multiple sub-Planckian axions
}

\author{
Kiwoon Choi, ${ }^{a, *}$ Hyungjin Kim, ${ }^{a, b, \dagger}$ and Seokhoon Yun ${ }^{a, b, \ddagger}$ \\ ${ }^{a}$ Center for Theoretical Physics of the Universe, \\ Institute for Basic Science (IBS), Daejeon 305-811, Korea \\ b Department of Phyiscs, KAIST, Daejeon 305-701, Korea
}

\begin{abstract}
We extend the Kim-Nilles-Peloso (KNP) alignment mechanism for natural inflation to models with $N>2$ axions, which obtains super-Planckian effective axion decay constant $f_{\text {eff }} \gg M_{P l}$ through an alignment of the anomaly coefficients of multiple axions having sub-Planckian fundamental decay constants $f_{0} \ll M_{P l}$. The original version of the KNP mechanism realized with two axions requires that some of the anomaly coefficients should be of the order of $f_{\text {eff }} / f_{0}$, which would be uncomfortably large if $f_{\text {eff }} / f_{0} \gtrsim \mathcal{O}(100)$ as suggested by the recent BICEP2 results. We note that the KNP mechanism can be realized with the anomaly coefficients of $\mathcal{O}(1)$ if the number of axions $N$ is large as $N \ln N \gtrsim 2 \ln \left(f_{\text {eff }} / f_{0}\right)$, in which case the effective decay constant can be enhanced as $f_{\text {eff }} / f_{0} \sim \sqrt{N !} n^{N-1}$ for $n$ denoting the typical size of the integer-valued anomaly coefficients. Comparing to the other multiple axion scenario, the $N$-flation scenario which requires $N \sim f_{\text {eff }}^{2} / f_{0}^{2}$, the KNP mechanism has a virtue of not invoking to a too large number of axions, although it requires a specific alignment of the anomaly coefficients, which can be achieved with a probability of $\mathcal{O}\left(f_{0} / f_{\text {eff }}\right)$ under a random choice of the anomaly coefficients. We also present a simple model realizing a multiple axion monodromy along the inflaton direction.
\end{abstract}

\footnotetext{
* kchoi@kaist.ac.kr

$\dagger$ hjkim06@kaist.ac.kr

‡yunsuhak@kaist.ac.kr
} 


\section{INTRODUCTION}

Recent detection of tensor modes in the cosmic microwave background by BICEP2 suggests that the inflation scale is rather high, $H_{I} \sim 10^{14} \mathrm{GeV}[1]$. In the context of slow roll inflation, such a high inflation scale implies that the inflaton field has experienced a super-Planckian excursion over the inflation period [2]. This poses a question if the inflaton field can be decoupled from high scale physics above the scale of quantum gravity, so that an effective field theory description of inflation does make sense.

An attractive solution to this puzzle is to introduce an approximate continuous shift symmetry along the inflaton direction. To implement this idea, in natural inflation [3] the inflaton is assumed to be a pseudo-Nambu-Goldstone boson $\phi$ with a periodic potential

$$
V(\phi)=\Lambda^{4}\left[1-\cos \left(\frac{\phi}{f}\right)\right],
$$

where $f$ is the axion decay constant which defines the fundamental domain of the axionlike inflaton field:

$$
\phi \equiv \phi+2 \pi f
$$

This inflaton potential is stable against perturbative quantum corrections, which can be assured through the approximate shift symmetry $\phi \rightarrow \phi+$ constant. Also, under a reasonable assumption on the nonperturbative dynamics generating the axion potential, one can justify that the above simple potential provides a good approximation to the full inflaton potential.

However, there is a difficulty in this simple setup. For successful slow roll inflation, the axion decay constant is required to have a super-Planckian value. The recent BICEP2 result makes the problem even more severe as it suggests [4]

$$
f \gtrsim 10 M_{P l}
$$

where $M_{P l} \simeq 2.4 \times 10^{18} \mathrm{GeV}$ is the reduced Planck mass. It appears to be difficult to get such a large axion decay constant from a sensible fundamental theory, particularly from string theory. In the limit where a controllable approximation is available, string theory predicts that the axion scale is typically around $g^{2} M_{P l} / 8 \pi^{2}[5]$.

During the past years, there have been several proposals to circumvent this problem. They include the two-axion model of Kim et al. [6], which obtains a super-Planckian effective 
axion decay constant through an alignment of the anomaly coefficients of two axions having sub-Planckian fundamental decay constants, a five-dimensional (5D) gauge-axion unification model in which the axionlike inflaton obtains a super-Planckian decay constant in the limit that 5D gauge coupling becomes weaker than the 5D gravitational coupling [7], a model with non-minimal axion-gravity coupling where gravitationally enhanced Hubble friction makes natural inflation operative with sub-Planckian axion decay constant [8], the $N$-flation scenario [9] based on the idea of assisted inflation [10] with many axions, and the axion monodromy based on either a string theoretic [11, 12] or field theoretic [13, 14] scheme for multiple windings in the axion field space. In this paper, we revisit the Kim-Nilles-Peloso (KNP) alignment mechanism to extend the scheme to models with $N>2$ axions.

The original version of the KNP mechanism realized with two axions requires that some of the anomaly coefficients should be of the order of $f_{\text {eff }} / f_{i}$, where $f_{\text {eff }} \gg M_{P l}$ is the superPlanckian effective decay constant of the axionic inflaton, while $f_{i} \ll M_{P l}$ are the subPlanckian fundamental axion decay constants in the model. In case that $f_{i} \sim g^{2} M_{P l} / 8 \pi^{2}$ [5] as suggested by string theory, this would require that some anomaly coefficients should be uncomfortably large as $f_{\text {eff }} / f_{i}=\mathcal{O}\left(10^{2}-10^{3}\right)$. We note that the KNP mechanism can be realized with the anomaly coefficients of $\mathcal{O}(1)$ if the number of axions is large as $N \ln N \gtrsim 2 \ln \left(f_{\text {eff }} / f_{i}\right)$, in which case the effective decay constant can be enhanced as $f_{\text {eff }} / f_{i} \sim$ $\sqrt{N !} n^{N-1}$ for $n$ denoting the typical size of the integer-valued anomaly coefficients. We examine also the probability for the KNP alignment to be achieved under a random choice of the anomaly coefficients.

In regard to enhancing the effective axion decay constant, a relevant question is how many fields do we need to get super-Planckian $f_{\text {eff }} \gg M_{P l}$. As the Planck scale receives a quadratically divergent radiative correction from each light field, schematically we have $\delta M_{P l}^{2} \propto N_{l} \Lambda^{2}$, where $N_{l}$ denotes the number of light fields and $\Lambda$ is the cutoff scale of loop momenta. Then the scheme would be in trouble if it requires a too large number of light fields as $N_{l} \geq f_{\text {eff }}^{2} / f_{i}^{2}$. In our multiple axion scenario, $f_{\text {eff }} / f_{i}$ grows exponentially as a function of $N$ for a fixed value of $n>1$, so the number of required axions is of the order of $\ln \left(f_{\text {eff }} / f_{i}\right)$. On the other hand, for the original two-axion KNP model [6], one needs $n=\mathcal{O}\left(f_{\text {eff }} / f_{i}\right)$, where the anomaly coefficient $n$ can be identified as the number of gaugecharged fermions generating the axion coupling to instantons. In the $N$-flation scenario [9], the number of required axions is $\mathcal{O}\left(f_{\text {eff }}^{2} / f_{i}^{2}\right)$. So our scheme can enhance $f_{\text {eff }}$ by introducing 
a parametrically smaller number of fields, as compared to the KNP two-axion model and the $N$-flation scenario.

In certain cases, the KNP mechanism can be interpreted as enhancing the effective axion decay constant as $f_{\text {eff }} / f_{i} \sim n \gg 1$ through the $Z_{n}$ monodromy structure of a light axion, which is induced along the inflaton direction by the mixing with heavy axions. In this

context, we present a simple model yielding $f_{\text {eff }} / f_{i} \sim \prod_{i=2}^{N} n_{i}$ through a multiple axion monodromy described by $\prod_{i=2}^{N} Z_{n_{i}}\left(n_{i}>1\right)$. We present also a model yielding $f_{\text {eff }} / f_{i} \sim 2^{N-1}$ even when all the integer-valued anomaly coefficients are restricted as $\left|n_{i j}\right| \leq 1$.

The organization of this paper is as follows. In Sec. III, we review the original two-axion model of KNP to illustrate the basic idea and set the notations. In Sec. III, we extend the KNP mechanism to models with $N>2$ axions. Sec. IV is the conclusion.

\section{KIM-NILLES-PELOSO MECHANISM WITH TWO AXIONS}

We begin with a brief review of the original Kim-Nilles-Peloso mechanism realized with two axions [6]. In the field basis of periodic axions:

$$
\phi_{i} \equiv \phi_{i}+2 \pi f_{i}
$$

the axion potential consistent with the axion periodicity is generically given by

$$
V\left(\phi_{i}\right)=\Lambda_{1}^{4}\left[1-\cos \left(\frac{n_{1} \phi_{1}}{f_{1}}+\frac{n_{2} \phi_{2}}{f_{2}}\right)\right]+\Lambda_{2}^{4}\left[1-\cos \left(\frac{m_{1} \phi_{1}}{f_{1}}+\frac{m_{2} \phi_{2}}{f_{2}}\right)\right],
$$

where $\vec{n}=\left(n_{1}, n_{2}\right)$ and $\vec{m}=\left(m_{1}, m_{2}\right)$ are linearly independent integer-valued coefficients, and $f_{i}(i=1,2)$ denote the fundamental axion decay constants which are presumed to be comparable to each other, while being significantly lower than the reduced Planck scale:

$$
f_{1} \sim f_{2} \ll M_{P l}
$$

Here we include only the leading nonperturbative effects generating the axion potential, under the assumption that the next order nonperturbative effects are small enough.

The integer-valued coefficients $n_{i}, m_{i}$ parametrize the discrete degrees of freedom in the underlying nonperturbative dynamics generating the axion potential. A simple possibility is that the axion potential is generated by hidden sector gauge field instantons through the symmetry breaking by anomalies. In such case, the model involves two non-Abelian hidden 
sector gauge groups $G_{a}(a=1,2)$, together with the gauge-charged fermions having the following couplings to axions:

$$
\sum_{I} \sum_{i} \lambda_{i I} f_{i} e^{i q_{i I} \phi_{i} / f_{i}} \bar{\psi}_{I L} \psi_{I R}+\text { H.c. }
$$

where $\lambda_{i I}$ denote dimensionless Yukawa couplings and $\psi_{I}$ are assumed to be charged Dirac fermions for simplicity. Then the Noether current of the nonlinearly realized Peccei-Quinn symmetries

$$
U(1)_{i}: \phi_{i} \rightarrow \phi_{i}+\alpha_{i} f_{i}, \quad \bar{\psi}_{I L} \psi_{I R} \rightarrow e^{-i q_{i I} \alpha_{i}} \bar{\psi}_{I L} \psi_{I R}
$$

have the $U(1)_{i}-G_{a^{-}} G_{a}$ anomalies as

$$
\partial_{\mu} J_{i}^{\mu}=\frac{n_{i}}{16 \pi^{2}} F_{1} \tilde{F}_{1}+\frac{m_{i}}{16 \pi^{2}} F_{2} \tilde{F}_{2}
$$

where $F_{a}$ are the gauge field strength of the gauge group $G_{a}$, and the anomaly coefficients are given by

$$
n_{i}=2 \sum_{I} q_{i I} \operatorname{Tr}\left(T_{1}^{2}\left(\psi_{I}\right)\right), \quad m_{i}=2 \sum_{I} q_{i I} \operatorname{Tr}\left(T_{2}^{2}\left(\psi_{I}\right)\right)
$$

for $T_{a}\left(\psi_{I}\right)(a=1,2)$ denoting the $G_{a}$-charge matrix of $\psi_{I}$ normalized as $\operatorname{Tr}\left(T_{a}^{2}\right)=1 / 2$ for the fundamental representation of $G_{a}$. With this symmetry breaking by anomalies, the gauge field instantons of $G_{a}$ generate the axion potential of the form (5). Based on this observation, in the following we will call $n_{i}, m_{i}$ the anomaly coefficients. However it should be noted that the axion potential (5) can be generated by different kinds of nonperturbative effects, for instance string theoretic instantons or hidden gaugino condensations. In such case, the integer coefficients $n_{i}, m_{i}$ can be determined by a variety of different discrete UV quantum numbers, e.g. the quantized fluxes, the number of stacked $D$-branes, and/or the number of windings for stringy instantons.

To discuss the KNP mechanism, it is convenient to consider the limit $\Lambda_{2}=0$, in which the axion potential is given by

$$
V\left(\phi_{i}\right)=\Lambda_{1}^{4}\left[1-\cos \left(\frac{n_{1} \phi_{1}}{f_{1}}+\frac{n_{2} \phi_{2}}{f_{2}}\right)\right] .
$$

Obviously this axion potential has a one-dimensional periodic flat direction in the twodimensional fundamental domain of the axion fields spanned by $\phi_{i}=\left[0,2 \pi f_{i}\right]$,

$$
\phi_{\text {flat }} \propto \frac{n_{2} \phi_{1}}{f_{2}}-\frac{n_{1} \phi_{2}}{f_{1}}
$$



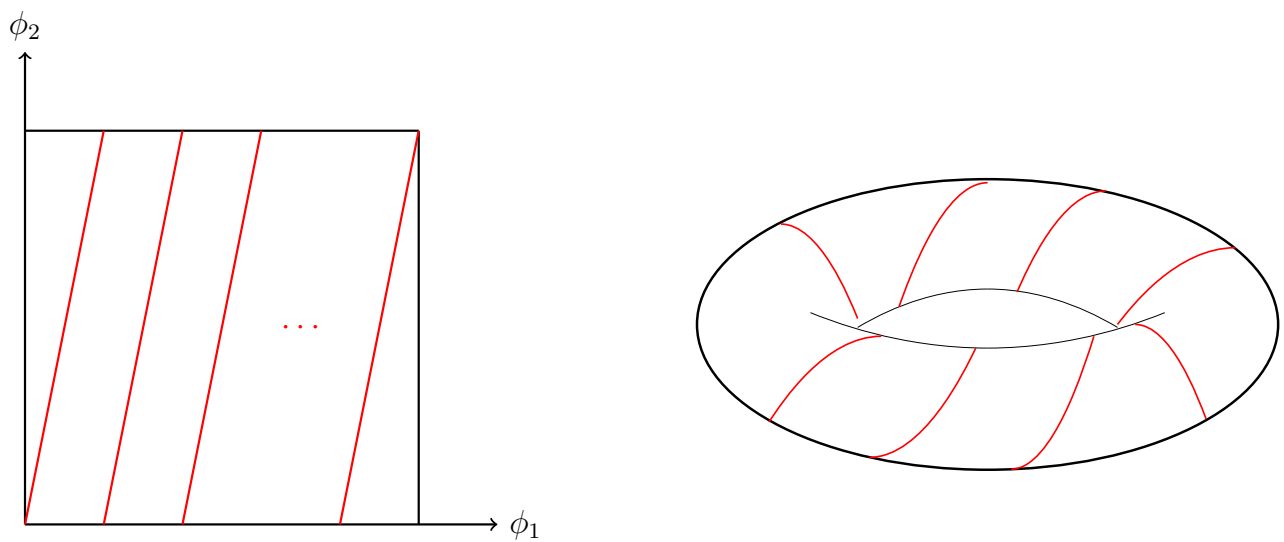

FIG. 1: Flat direction in the fundamental domain of axion fields in the limit $\Lambda_{2}=0$. Even though the fundamental domain is sub-Planckian with $f_{i} \ll M_{P l}$, the flat direction can have a super-Planckian length if one (or both) of $n_{i} / \operatorname{gcd}\left(n_{1}, n_{2}\right)$ is large enough. The right panel depicts the flat direction in the fundamental domain for which the axion periodicity is manifest.

which can be identified as the inflaton direction. One easily finds that the length of this periodic flat direction is given by

$$
\ell_{\text {flat }}=\frac{2 \pi \sqrt{n_{1}^{2} f_{2}^{2}+n_{2}^{2} f_{1}^{2}}}{\operatorname{gcd}\left(n_{1}, n_{2}\right)}
$$

where gcd $\left(n_{1}, n_{2}\right)$ denotes the greatest common divisor of $n_{1}$ and $n_{2}$. This shows that a super-Planckian flat direction with $\ell_{\text {flat }} \gg M_{P l} \gg f_{i}$ can be developed on the twodimensional sub-Planckian domain if

$$
\frac{n_{1}}{\operatorname{gcd}\left(n_{1}, n_{2}\right)} \text { or } \frac{n_{2}}{\operatorname{gcd}\left(n_{1}, n_{2}\right)} \gg \frac{M_{P l}}{f_{i}} \gg 1
$$

In Fig. 1, we depict the flat direction in the fundamental domain of axion fields, which has a length given by (12). Since the axionic inflaton of natural inflation rolls down along this periodic flat direction, its effective decay constant is bounded as

$$
f_{\text {eff }} \leq \frac{\ell_{\text {flat }}}{2 \pi}
$$

which means that at least one of $n_{i}$ should be as large as $\operatorname{gcd}\left(n_{1}, n_{2}\right) f_{\text {eff }} / f_{i}$.

Turning on the second axion potential

$$
\Delta V=\Lambda_{2}^{4}\left[1-\cos \left(\frac{m_{1} \phi_{1}}{f_{1}}+\frac{m_{2} \phi_{2}}{f_{2}}\right)\right],
$$

a nontrivial potential is developed along the periodic flat direction having a length (12). Even when $\ell_{\text {flat }} \gg M_{P l}$, natural inflation is not guaranteed as the inflaton potential induced 
by $\Delta V$ generically has multiple modulations along the flat direction. We find that the number of modulations over the full range of the periodic flat direction is given by

$$
N_{\text {mod }}=\frac{\left|n_{1} m_{2}-n_{2} m_{1}\right|}{\operatorname{gcd}\left(n_{1}, n_{2}\right)}
$$

which results in the effective decay constant

$$
f_{\text {eff }}=\frac{\ell_{\text {flat }}}{2 \pi N_{\text {mod }}}=\frac{\sqrt{n_{1}^{2} f_{2}^{2}+n_{2}^{2} f_{1}^{2}}}{\left|n_{1} m_{2}-n_{2} m_{1}\right|} .
$$

It is straightforward to derive the above form of the effective axion decay constant [6]. Taking the rotated axion field basis:

$$
\psi=\frac{n_{1} f_{2} \phi_{1}+n_{2} f_{1} \phi_{2}}{\sqrt{n_{1}^{2} f_{2}^{2}+n_{2}^{2} f_{1}^{2}}}, \quad \phi_{\text {flat }}=\frac{n_{2} f_{1} \phi_{1}-n_{1} f_{2} \phi_{2}}{\sqrt{n_{1}^{2} f_{2}^{2}+n_{2}^{2} f_{1}^{2}}},
$$

the axion potential (5) can be written as

$$
V\left(\psi, \phi_{\text {flat }}\right)=\Lambda_{1}^{4}\left[1-\cos \left(\frac{\psi}{f_{\psi}}\right)\right]+\Lambda_{2}^{4}\left[1-\cos \left(\frac{\psi}{f_{\psi}^{\prime}}+\frac{\phi_{\text {flat }}}{f_{\text {eff }}}\right)\right],
$$

where

$$
\begin{aligned}
f_{\psi} & =\frac{f_{1} f_{2}}{\sqrt{n_{1}^{2} f_{2}^{2}+n_{2}^{2} f_{1}^{2}}}, \\
f_{\psi}^{\prime} & =\frac{f_{1} f_{2} \sqrt{n_{1}^{2} f_{2}^{2}+n_{2}^{2} f_{1}^{2}}}{n_{1} m_{1} f_{2}^{2}+n_{2} m_{2} f_{1}^{2}} \\
f_{\mathrm{eff}} & =\frac{\sqrt{n_{1}^{2} f_{2}^{2}+n_{2}^{2} f_{1}^{2}}}{\left|n_{1} m_{2}-n_{2} m_{1}\right|}
\end{aligned}
$$

Taking the limit $\Lambda_{1}^{4} \gg \Lambda_{2}^{4}$, the heavy field component $\psi$ can be integrated out, yielding the effective potential of the light inflaton direction $\phi_{\text {flat }}$ as

$$
V_{\text {eff }}\left(\phi_{\text {flat }}\right)=\Lambda_{2}^{4}\left[1-\cos \left(\frac{\phi_{\text {flat }}}{f_{\text {eff }}}\right)\right] .
$$

From the above expression of $f_{\text {eff }}$, it is clear that $f_{\text {eff }} \gg f_{i}$ is not a generic feature of the model, but requires a special alignment of the anomaly coefficients $n_{i}, m_{i}$. Note that in the absence of any alignment, generically

$$
f_{\text {eff }} \sim \frac{f_{i}}{n}
$$

where $n$ represents the typical size of the anomaly coefficients $n_{i}, m_{i}$. Obviously there can be many different choices (or alignments) of the anomaly coefficients yielding $f_{\text {eff }} / f_{i} \gg 1$. A 
simple possibility is that one or both of $n_{i}$ are large, while the denominator $\left(n_{1} m_{2}-n_{2} m_{1}\right)$ is tuned to be $\mathcal{O}(1)$. As a specific example, KNP considered the case that $n_{2} \gg 1$ and the other three anomaly coefficients are given by $m_{1}=n_{1}=1$ and $m_{2}=n_{2}+\mathcal{O}(1)$ [6]. Of course, one can consider different examples as we do below, for instance $n_{2} \gg 1$ with $n_{1}=m_{2}=1, m_{1}=0$.

To understand the geometric meaning of the required alignment, one can introduce an alignment angle $\delta \theta$ as

$$
\sin \delta \theta \equiv \frac{\left|n_{1} m_{2}-m_{1} n_{2}\right|}{\sqrt{\left(n_{1}^{2}+n_{2}^{2}\right)\left(m_{1}^{2}+m_{2}^{2}\right)}},
$$

and rewrite the effective decay constant as

$$
f_{\mathrm{eff}}=\frac{1}{\sin \delta \theta}\left(\frac{n_{1}^{2} f_{2}^{2}+n_{2}^{2} f_{1}^{2}}{\left(n_{1}^{2}+n_{2}^{2}\right)\left(m_{1}^{2}+m_{2}^{2}\right)}\right)^{1 / 2} .
$$

Note that $\delta \theta$ corresponds to the angle between the heavy field direction $\psi$ and the other field direction $\phi$ of the second axion potential $\Delta V$ :

$$
\psi \propto \frac{n_{1} \phi_{1}}{f_{1}}+\frac{n_{2} \phi_{2}}{f_{2}}, \quad \phi \propto \frac{m_{1} \phi_{1}}{f_{1}}+\frac{m_{2} \phi_{2}}{f_{2}} .
$$

For the case of two axions, one of the economic ways to get $f_{\text {eff }} / f_{i} \gg 1$ is to have

$$
n_{1} \sim n_{2}=\mathcal{O}\left(f_{\text {eff }} / f_{i}\right), \quad m_{1} \sim m_{2}=\mathcal{O}(1),
$$

for which

$$
\delta \theta=\mathcal{O}\left(f_{i} / f_{\text {eff }}\right)
$$

This implies that the probability for achieving $f_{\text {eff }} \gg f_{i}$ under a random choice of the anomaly coefficients, but within the specific region of (25), is given by

$$
P\left(f_{\text {eff }} / f_{i}\right)=\mathcal{O}\left(f_{i} / f_{\text {eff }}\right)
$$

On the other hand, if one extends the random choice to the generic region where all the anomaly coefficients can be of the order of $f_{\text {eff }} / f_{i}$, one needs an alignment angle $\delta \theta=$ $\mathcal{O}\left(f_{i}^{2} / f_{\text {eff }}^{2}\right)$, and then the probability for achieving $f_{\text {eff }} \gg f_{i}$ is reduced as

$$
P\left(f_{\text {eff }} / f_{i}\right)=\mathcal{O}\left(f_{i}^{2} / f_{\text {eff }}^{2}\right) .
$$


A particularly interesting choice [15, 16] of the anomaly coefficients realizing the KNP alignment is

$$
n_{1}=m_{2}=1, \quad m_{1}=0, \quad\left|n_{2}\right| \gg 1,
$$

for which the light inflaton direction is identified as

$$
\phi_{\text {flat }}=\frac{n_{2} f_{1} \phi_{1}-f_{2} \phi_{2}}{f_{\text {eff }}} \simeq \phi_{1}
$$

with the effective decay constant

$$
f_{\mathrm{eff}}=\sqrt{n_{2}^{2} f_{1}^{2}+f_{2}^{2}} \simeq\left|n_{2}\right| f_{1}
$$

where we assumed $f_{1} \sim f_{2}$. In this case, we have

$$
e^{i \phi_{1} / f_{1}}=e^{-i n_{2} \phi_{2} / f_{2}}
$$

along the inflaton direction. Then the enhanced effective axion decay constant can be considered as a consequence of the $Z_{n_{2}}$ monodromy structure of $\phi_{1}$, which is induced by the mixing with the heavy axion component $\phi_{2}$.

\section{GENERALIZATION TO $N>2$ AXIONS}

It is in fact straightforward to generalize the KNP mechanism to the case with $N>2$ axions. ${ }^{1}$ In the presence of $N$ axions, again in the periodic axion field basis

$$
\phi_{i} \equiv \phi_{i}+2 \pi f_{i} \quad(i=1,2, \ldots, N)
$$

the axion potential takes the form

$$
V=\sum_{i=1}^{N} \Lambda_{i}^{4}\left[1-\cos \left(\sum_{j=1}^{N} \frac{n_{i j} \phi_{j}}{f_{j}}\right)\right],
$$

where $\overrightarrow{n_{i}}=\left(n_{i 1}, n_{i 2}, . ., n_{i N}\right)$ are linearly independent integer-valued anomaly coefficients, and $f_{i} \ll M_{P l}$ are the fundamental decay constants which are presumed to be comparable to each other. To proceed, let us take the limit

$$
\Lambda_{N}=0
$$

\footnotetext{
1 A generalization to the case with $N>2$ axions has been discussed in [17] to accommodate the intermediate scale QCD axion as well as a quintessence axion with Planck scale decay constant in the model.
} 
for which the one-dimensional periodic flat direction is parametrized as

$$
\begin{aligned}
\phi_{\text {flat }} & \propto \sum_{i} X_{i} \phi_{i} \\
& \equiv \operatorname{Det}\left(\begin{array}{cccc}
\phi_{1} & \phi_{2} & \cdots & \phi_{N} \\
\frac{n_{11}}{f_{1}} & \frac{n_{12}}{f_{2}} & \cdots & \frac{n_{1 N}}{f_{N}} \\
\vdots & \vdots & & \vdots \\
\frac{n_{N-1,1}}{f_{1}} & \frac{n_{N-1,2}}{f_{2}} & \cdots & \frac{n_{N-1, N}}{f_{N}}
\end{array}\right),
\end{aligned}
$$

where

$$
X_{i}=\frac{C_{i} f_{i}}{\prod_{j} f_{j}}
$$

for

$$
C_{i}=(-1)^{i+1} \operatorname{Det}\left(\begin{array}{ccccccc}
n_{11} & n_{12} & \ldots & n_{1,(i-1)} & n_{1,(i+1)} & \ldots & n_{1 N} \\
n_{21} & n_{22} & \ldots & n_{2,(i-1)} & n_{2,(i+1)} & \ldots & n_{2 N} \\
\vdots & \vdots & & \vdots & \vdots & & \vdots \\
n_{N-1,1} & n_{N-1,2} & \ldots & n_{N-1,(i-1)} & n_{N-1,(i+1)} & \ldots & n_{N-1, N}
\end{array}\right)
$$

The length of this periodic flat direction is determined by the minimal discrete shift $\Delta \phi_{i}$ along the flat direction, under which the axion field configuration comes back to the original configuration. One then finds

$$
\Delta \phi_{i}=\frac{2 \pi C_{i} f_{i}}{\operatorname{gcd}\left(C_{1}, C_{2}, \cdots, C_{N}\right)}
$$

yielding the length of the flat direction:

$$
\ell_{\text {flat }}=\frac{2 \pi \sqrt{\sum_{i=1}^{N} C_{i}^{2} f_{i}^{2}}}{\operatorname{gcd}\left(C_{1}, C_{2}, \cdots, C_{N}\right)},
$$

where gcd $\left(C_{1}, C_{2}, \cdots, C_{N}\right)$ denotes the greatest common divisor of all $C_{i}$.

For generic anomaly coefficients $n_{i j}$, the magnitude of $C_{i}$ is quite sensitive to the number of axions, as well as to the typical size of $n_{i j}$. Here we are interested in the limit $N \gg 1$ with

$$
\left|n_{i j}\right| \leq n_{\max }=\mathcal{O}(\text { few })
$$

To proceed, we can regard $n_{i j}$ as a random integer-valued variable with flat probability distribution:

$$
P\left(n_{i j}\right)=\frac{1}{2 n_{\max }+1}
$$


We then have

$$
\begin{aligned}
& \left\langle n_{i j}\right\rangle=\sum_{n_{i j}=-n_{\max }}^{n_{\max }} n_{i j} P\left(n_{i j}\right)=0, \\
& \left\langle n_{i j}^{2}\right\rangle=\sum_{n_{i j}=-n_{\max }}^{n_{\max }} n_{i j}^{2} P\left(n_{i j}\right)=\frac{1}{3} n_{\max }\left(1+n_{\max }\right) \equiv n^{2},
\end{aligned}
$$

where

$$
n=\sqrt{n_{\max }\left(1+n_{\max }\right) / 3}
$$

denotes the typical size of the anomaly coefficients in the range (40). One can similarly compute the expectation value of $C_{i}^{2}$. For $C_{N}=\sum_{\sigma} \operatorname{sgn}(\sigma) n_{1 \sigma(1)} \cdots n_{(N-1) \sigma(N-1)}$, where the summation is over all possible permutations of $\{1,2, \ldots, N-1\}$, one easily finds

$$
\left\langle C_{N}^{2}\right\rangle=\left\langle\sum_{\sigma}\left(n_{1 \sigma(1)} \cdots n_{(N-1) \sigma(N-1)}\right)^{2}\right\rangle=(N-1) ! n^{2(N-1)},
$$

and therefore

$$
\left\langle\sum_{i=1}^{N} C_{i}^{2}\right\rangle=N ! \cdot n^{2(N-1)}
$$

As implied by this expectation value, in most cases we have ${ }^{2}$

$$
\left(\sum_{i=1}^{N} C_{i}^{2}\right)^{1 / 2} \sim \sqrt{N !} n^{N-1}, \quad \operatorname{gcd}\left(C_{1}, C_{2}, \ldots, C_{N}\right)=\mathcal{O}(1)
$$

and therefore a flat direction enhanced as

$$
\frac{\ell_{\text {flat }}}{2 \pi f_{i}} \sim \sqrt{N !} n^{N-1}
$$

where the sub-Planckian axion decays constants $f_{i}$ are assumed to be comparable to each other. Here we are interested in the case that the sub-Planckian axions $\phi_{i}$ originate from higher-dimensional tensor gauge fields in compactified string theory, in which case all $f_{i}$ are comparable to $M_{s t} / 8 \pi^{2}$ for the string scale $M_{s t}[5]$. Note that the flat direction length is exponentially enhanced in the limit $N \gg 1$ when the typical anomaly coefficient $n>1$. As

\footnotetext{
${ }^{2}$ We have in fact an upper bound $\sqrt{\sum_{i} C_{i}^{2}}<N^{N / 2} n_{\max }^{N-1}$. We found through a numerical analysis that $\sqrt{\sum_{i} C_{i}^{2}} \gtrsim 0.2 \sqrt{N !} n^{N-1}$ in most cases of our interest. It is also known that a probability for $\operatorname{gcd}\left(C_{i}\right)=1$ under a random choice of $C_{i}$ within the range $\left|C_{i}\right| \leq Q$ is given by $P\left(\operatorname{gcd}\left(C_{i}\right)=1\right)=1 / \zeta(N)+\mathcal{O}(1 / Q)$, which is close to the unity in the limit $N \gg 1$ and $Q \gg 1$. Although in our case $C_{i}$ is not a randomly chosen integer, but a determinant of the randomly chosen anomaly coefficients $n_{i j}$, we confirmed again through a numerical analysis that $\operatorname{gcd}\left(C_{i}\right)=1$ in most cases of our interest.
} 
a result, an exponentially long flat direction can be obtained with the anomaly coefficients $\left|n_{i j}\right| \leq n_{\max }=\mathcal{O}$ (few), with a moderately large number of axions

$$
N \ln N \sim 2 \ln \left(\ell_{\text {flat }} / 2 \pi f_{i}\right)
$$

This can be understood by noting that the flat direction generically winds each of the additional axion dimensions by about $n$ times, which would explain the factor $n^{N-1}$, and there are also combinatoric degrees of freedom for the windings of the $N$-dimensional torus of axion fields, which would explain the factor $\sqrt{N !}$.

Introducing the last part of the axion potential

$$
\Delta V=\Lambda_{N}^{4}\left[1-\cos \left(\sum_{j} \frac{n_{N j} \phi_{j}}{f_{j}}\right)\right],
$$

a periodic potential is developed along the flat direction. Again super-Planckian flat direction does not guarantee a super-Planckian effective decay constant. Instead we have

$$
f_{\text {eff }}=\frac{\ell_{\text {flat }}}{2 \pi N_{\text {mod }}}
$$

where $N_{\text {mod }}$ is the number of modulations of the axion potential over the full period of the flat direction. Taking the limit $\Lambda_{i} \gg \Lambda_{N}(i=1,2, \ldots, N-1)$ to integrate out the $(N-1)$ heavy axions, we find that the effective potential of the flat direction is given by

$$
V_{\text {eff }}\left(\phi_{\text {flat }}\right)=\Lambda_{N}^{4}\left[1-\cos \left(\frac{\phi_{\text {flat }}}{f_{\text {eff }}}\right)\right],
$$

where

$$
f_{\mathrm{eff}}=\frac{\sqrt{\sum_{i=1}^{N} C_{i}^{2} f_{i}^{2}}}{|\operatorname{Det} \mathbb{N}|}
$$

for

$$
\operatorname{Det} \mathbb{N}=\operatorname{Det}\left(\begin{array}{cccc}
n_{N 1} & n_{N 2} & \cdots & n_{N N} \\
n_{11} & n_{12} & \cdots & n_{1 N} \\
\vdots & \vdots & & \vdots \\
n_{(N-1) 1} & n_{(N-1) 2} & \cdots & n_{(N-1) N}
\end{array}\right)=\sum_{i} C_{i} n_{N i} \text {. }
$$

This tells that the number of modulations over the full range of the flat direction is

$$
N_{\text {mod }}=\frac{\left|\sum_{i} C_{i} n_{N i}\right|}{\operatorname{gcd}\left(C_{1}, C_{2}, \cdots, C_{N}\right)} .
$$


To justify our approach to integrate out the $(N-1)$ heavy axions, let us briefly examine the axion masses in our scheme. For the axion potential (34), the $N \times N$ axion mass-square matrix is given by $m_{k l}^{2}=\sum_{i} \Lambda_{i}^{4} n_{i k} n_{i l} / f_{k} f_{l}$, yielding

$$
\operatorname{Det} m^{2}=(\operatorname{Det} \mathbb{N})^{2} \prod_{i}^{N} \frac{\Lambda_{i}^{4}}{f_{i}^{2}}
$$

In the presence of light flat direction, its mass square is given by

$$
m_{\text {flat }}^{2} \simeq \frac{\Lambda_{N}^{4}}{f_{\text {eff }}^{2}}=\frac{\Lambda_{N}^{4}(\operatorname{Det} \mathbb{N})^{2}}{\sum_{j} C_{j}^{2} f_{j}^{2}} .
$$

Then the mass-square determinant can be written as

$$
\begin{aligned}
\operatorname{Det} m^{2} & =(\operatorname{Det} \mathbb{N})^{2} \prod_{i}^{N} \frac{\Lambda_{i}^{4}}{f_{i}^{2}}=\frac{\Lambda_{N}^{4}}{f_{\text {eff }}^{2}} \frac{f_{\text {eff }}^{2}}{f_{N}^{2}}(\operatorname{Det} \mathbb{N})^{2} \prod_{i}^{N-1} \frac{\Lambda_{i}^{4}}{f_{i}^{2}} \\
& =m_{\text {flat }}^{2} \frac{\sum C_{j}^{2} f_{j}^{2}}{f_{N}^{2}} \prod_{i}^{N-1} \frac{\Lambda_{i}^{4}}{f_{i}^{2}} \sim m_{\text {flat }}^{2} \prod_{i}^{N-1} \frac{N n^{2} \Lambda_{i}^{4}}{e f_{i}^{2}} \sim m_{\text {flat }}^{2} \prod_{i}^{N-1} m_{\text {heavy }}^{2}(i),
\end{aligned}
$$

where $m_{\text {heavy }}(i)$ is the $i$ th heavy axion mass, and we have used $\sum_{j} C_{j}^{2} f_{j}^{2} / f_{N}^{2} \sim N ! n^{2(N-1)} \sim$ $N^{N} n^{2(N-1)} / e^{N}$ under the assumption that all $f_{i}$ are comparable to each other. We then find

$$
\frac{m_{\text {heavy }}^{2}(i)}{m_{\text {flat }}^{2}} \sim \frac{N n^{2}}{e} \frac{f_{\text {eff }}^{2}}{f_{i}^{2}} \frac{\Lambda_{i}^{4}}{\Lambda_{N}^{4}}
$$

which shows that the heavy axions are heavy enough compared to the flat direction if the anomaly coefficients are aligned to yield $f_{\text {eff }} \gg f_{i}$, even when $\Lambda_{N}$ is comparable to $\Lambda_{i}$ $(i=1, \ldots, N-1)$.

As in the case with two axions, it is clear that $f_{\text {eff }} \gg f_{i}$ is not a generic feature, but requires a specific alignment of the anomaly coefficients. Yet, compared to the case of two axions, a notable difference is that the mechanism does not require large anomaly coefficients of $\mathcal{O}\left(f_{\text {eff }} / f_{i}\right)$, but a moderately large number of axions $N \ln N \gtrsim 2 \ln \left(f_{\text {eff }} / f_{i}\right)$ together with the anomaly coefficients $n_{i j}=\mathcal{O}(1)$. To quantify the required degree of alignment, let us introduce an alignment angle as in the case of two axions:

$$
\sin \delta \theta \equiv \frac{\left|\vec{C} \cdot \vec{n}_{N}\right|}{|\vec{C}|\left|\vec{n}_{N}\right|}=\frac{|\operatorname{Det} \mathbb{N}|}{|\vec{C}|\left|\vec{n}_{N}\right|} .
$$

Then the effective decay constant reads as

$$
f_{\mathrm{eff}}=\frac{1}{\sin \delta \theta} \frac{\sqrt{\sum_{i=1}^{N} C_{i}^{2} f_{i}^{2}}}{|\vec{C}|\left|\vec{n}_{N}\right|} \sim \frac{f_{i}}{\left|\vec{n}_{N}\right| \sin \delta \theta},
$$


implying that we need to align $\delta \theta$ to be small as

$$
\delta \theta=\mathcal{O}\left(f_{i} / f_{\text {eff }}\right)
$$

This also suggests that the probability for having $f_{\text {eff }} \gg f_{i}$ under a random choice of the anomaly coefficients in the range $\left|n_{i j}\right| \leq n_{\max }=\mathcal{O}(1)$ is given by

$$
P\left(f_{\text {eff }} / f_{i}\right)=\mathcal{O}\left(f_{i} / f_{\text {eff }}\right)
$$

Note that in the case of two axions we have a similar probability only when the random selection is limited to a specific (economic) region of the anomaly coefficients, for instance the region of (25), while $P\left(f_{\text {eff }} / f_{i}\right)=\mathcal{O}\left(f_{i}^{2} / f_{\text {eff }}^{2}\right)$ if one extends the random selection to the general region where all the anomaly coefficients can be comparable to each other.

In view of that the fundamental axion decay constants in string theory are typically in the range $f_{i} \sim 10^{16}-10^{17} \mathrm{GeV}$ [5], while a successful natural inflation compatible with the recent BICEP2 results [1] requires $f_{\text {eff }} \gtrsim 10 M_{P l}$, we are particularly interested in the minimal number of axions which can yield

$$
f_{\text {eff }} / f_{i}=\mathcal{O}\left(10^{2}-10^{3}\right) \text { for }\left|n_{i j}\right| \leq n_{\max }\left(n_{\max }=1,2,3\right)
$$

According to our discussion above, the corresponding range of $N$ is roughly given by

$$
N \ln N \gtrsim 2 \ln \left(f_{\text {eff }} / f_{i}\right)
$$

We have performed a numerical analysis to evaluate $P\left(f_{\text {eff }} / f_{i}\right)$ for the three different values of $f_{\text {eff }} / f_{i}$ :

$$
f_{\text {eff }} / f_{i}=10^{2}, 5 \times 10^{2}, 10^{3}
$$

when

$$
N=8-15\left(n_{\max }=1\right), \quad N=7-14\left(n_{\max }=2\right), \quad N=5-12\left(n_{\max }=3\right) .
$$

The results are depicted in Table I, which confirms that the probability for the necessary alignment to be achieved under a random choice of the anomaly coefficients is indeed of the order of $f_{i} / f_{\text {eff }}$.

Before closing this section, let us present a couple of explicit models which achieve an exponentially enhanced effective axion decay constant within the framework discussed above. 


\begin{tabular}{|c|c|c|c|c|c|c|c|c|c|c|c|}
\hline \multirow{2}{*}{$N$} & \multicolumn{3}{|c|}{$f_{\text {eff }} / f_{i}$} & \multirow{2}{*}{$N$} & \multicolumn{3}{|c|}{$f_{\text {eff }} / f_{i}$} & \multirow{2}{*}{$N$} & \multicolumn{3}{|c|}{$f_{\text {eff }} / f_{i}$} \\
\hline & 100 & 500 & 1000 & & 100 & 500 & 1000 & & 100 & 500 & 1000 \\
\hline 8 & 0.009 & 0. & 0. & 7 & 0.216 & 0.030 & 0. & 5 & 0.060 & 0. & 0. \\
\hline 9 & 0.064 & 0. & 0. & 8 & 0.411 & 0.210 & 0.05 & 6 & 0.202 & 0.060 & 0. \\
\hline 10 & 0.258 & 0.020 & 0. & 9 & 0.466 & 0.530 & 0.26 & 7 & 0.322 & 0.230 & 0.09 \\
\hline 11 & 0.487 & 0.105 & 0.01 & 10 & 0.542 & 0.445 & 0.43 & 8 & 0.373 & 0.225 & 0.31 \\
\hline 12 & 0.707 & 0.275 & 0.12 & 11 & 0.512 & 0.470 & 0.70 & 9 & 0.383 & 0.370 & 0.40 \\
\hline 13 & 0.797 & 0.500 & 0.41 & 12 & 0.519 & 0.660 & 0.64 & 10 & 0.393 & 0.390 & 0.30 \\
\hline 14 & 0.938 & 0.800 & 0.56 & 13 & 0.585 & 0.585 & 0.47 & 11 & 0.408 & 0.370 & 0.41 \\
\hline 15 & 0.855 & 0.850 & 0.77 & 14 & 0.530 & 0.490 & 0.43 & 12 & 0.404 & 0.355 & 0.39 \\
\hline
\end{tabular}

TABLE I: The probability to $f_{i} / f_{\text {eff }}$ ratio, $R=P /\left(f_{i} / f_{\text {eff }}\right)$, for the necessary alignment under $10^{5}$ random choices of the anomaly coefficients. We have considered $N=8-15$ for $n_{\max }=1$, $N=7-14$ for $n_{\max }=2$, and $N=5-12$ for $n_{\max }=3$.

Our first model is

$$
\begin{aligned}
V & =\Lambda_{1}^{4}\left[1-\cos \left(\frac{\phi_{1}}{f_{1}}+\frac{n_{2} \phi_{2}}{f_{2}}\right)\right]+\Lambda_{2}^{4}\left[1-\cos \left(\frac{\phi_{2}}{f_{2}}+\frac{n_{3} \phi_{3}}{f_{3}}\right)\right]+\cdots \\
& +\Lambda_{N-1}^{4}\left[1-\cos \left(\frac{\phi_{N-1}}{f_{N-1}}+\frac{n_{N} \phi_{N}}{f_{N}}\right)\right]+\Lambda_{N}^{4}\left[1-\cos \left(\frac{\phi_{N}}{f_{N}}\right)\right]
\end{aligned}
$$

which is designed to realize a multiple axion monodromy along the inflaton direction. The anomaly coefficient matrix of the model takes the form

$$
\mathbb{N}=\left(n_{i j}\right)=\left(\begin{array}{ccccc}
1 & n_{2} & & & \\
& 1 & n_{3} & & \\
& & 1 & \ddots & \\
& & & \ddots & n_{N} \\
& & & & 1
\end{array}\right)
$$

for which

$$
\operatorname{Det} \mathbb{N}=1, \quad\left|C_{i}\right|=\prod_{j=i+1}^{N} n_{j}
$$

The resulting effective axion decay constant is given by

$$
f_{\text {eff }}=\frac{\sqrt{\sum_{i=1}^{N} f_{i}^{2} C_{i}^{2}}}{\operatorname{Det} \mathbb{N}}=\left(\sum_{i=1}^{N} \prod_{j=i+1}^{N} n_{j}^{2} f_{i}^{2}\right)^{1 / 2} \sim n_{2} n_{3} \cdots n_{N} f_{1}
$$




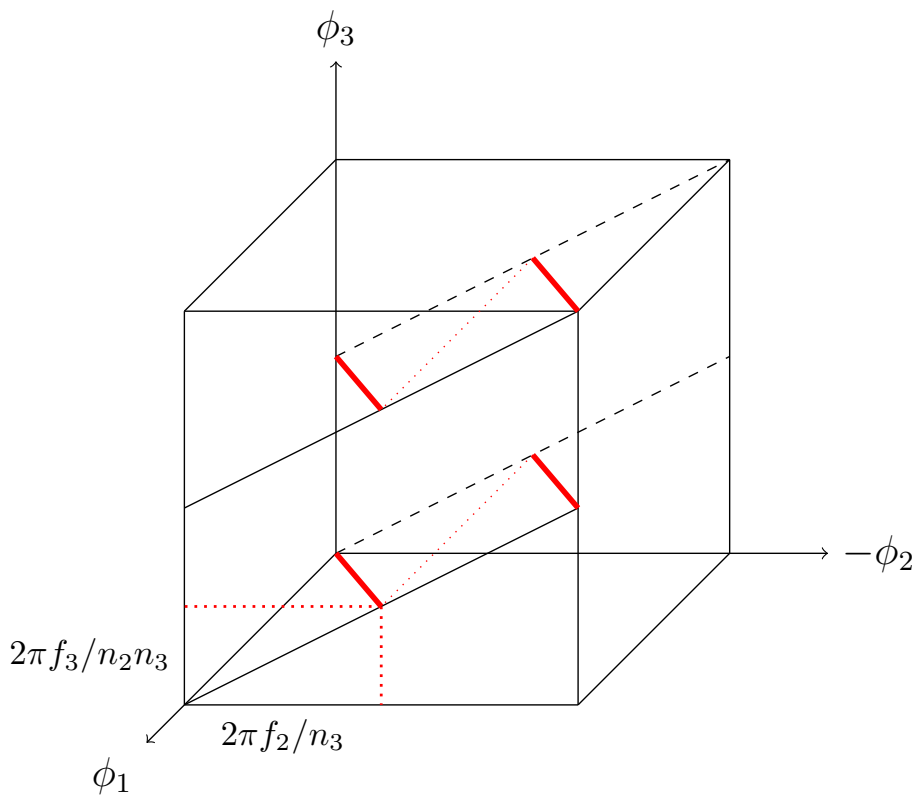

FIG. 2: Multiple monodromy structure for the three-axion model with $n_{2}=n_{3}=2$. The solid red line represents the inflaton direction in the fundamental domain of three axions. Note that $\Delta \phi_{2}=$ $2 \pi f_{2}$ along the inflaton direction requires $\Delta \phi_{1}=2 \pi n_{2} f_{1}$, and $\Delta \phi_{3}=2 \pi f_{3}$ requires $\Delta \phi_{2}=2 \pi n_{3} f_{2}$. As a result, $\Delta \phi_{3}=2 \pi f_{3}$ along the inflaton direction yields $\Delta \phi_{1}=2 \pi n_{2} n_{3} f_{1}$.

if $n_{i}>1$ and $f_{1} \sim f_{2} \sim \cdots \sim f_{N}$. In the limit $\Lambda_{N} \ll \Lambda_{i}(i=1, \ldots, N-1)$, the inflaton direction is determined to be

$$
\phi_{\text {flat }} \propto \sum_{i} C_{i} \phi_{i}=\left(\prod_{i=2}^{N} n_{i}\right) \phi_{1}-\left(\prod_{i=3}^{N} n_{i}\right) \phi_{2}+\cdots-n_{N} \phi_{N-1}+\phi_{N}
$$

This model can be considered as a generalization of the two axion models of [15, 16], and realizes a multiple axion monodromy $\prod_{i=2}^{N} Z_{n_{i}}$ along the inflaton direction. As a consequence, in order for the $N$ th axion $\phi_{N}$ to travel one period along the inflaton direction, i.e. $\Delta \phi_{N}=2 \pi f_{N}$, the other axions $\phi_{i}(i=1,2, \ldots, N-1)$ should experience a multiple winding as

$$
\frac{\Delta \phi_{i}}{2 \pi f_{i}}=\prod_{j \geq i+1}^{N} n_{j}
$$

In Fig. 2, we depict such multiple monodromy structure for the case of $N=3$ and $n_{2}=$ $n_{3}=2$. 
Our second model is

$$
\begin{aligned}
V & =\Lambda_{1}^{4}\left[1-\cos \left(\frac{\phi_{1}}{f_{1}}+\frac{\phi_{2}}{f_{2}}-\frac{\phi_{3}}{f_{3}}+\cdots+(-)^{N} \frac{\phi_{N}}{f_{N}}\right)\right] \\
& +\Lambda_{2}^{4}\left[1-\cos \left(\frac{\phi_{2}}{f_{2}}+\frac{\phi_{3}}{f_{3}}-\frac{\phi_{4}}{f_{4}}+\cdots+(-)^{N-1} \frac{\phi_{N}}{f_{N}}\right)\right]+\cdots \\
& +\Lambda_{N-2}^{4}\left[1-\cos \left(\frac{\phi_{N-2}}{f_{N-2}}+\frac{\phi_{N-1}}{f_{N-1}}-\frac{\phi_{N}}{f_{N}}\right)\right] \\
& +\Lambda_{N-1}^{4}\left[1-\cos \left(\frac{\phi_{N-1}}{f_{N-1}}+\frac{\phi_{N}}{f_{N}}\right)\right]+\Lambda_{N}^{4}\left[1-\cos \left(\frac{\phi_{N}}{f_{N}}\right)\right],
\end{aligned}
$$

which is designed to achieve an exponentially enhanced effective decay constant even when all the integer-valued anomaly coefficients are restricted as $\left|n_{i j}\right| \leq 1$. In this case, we have

$$
\operatorname{Det} \mathbb{N}=1, \quad C_{i}^{2}=4^{(N-1-i)} \quad(i=1,2, \cdots, N-1), \quad C_{N}^{2}=1
$$

Then, assuming that all fundamental decay constants are comparable to each other, the effective decay constant is exponentially enhanced as

$$
f_{\text {eff }} \sim \sqrt{\frac{1}{3}\left(4^{N-1}+2\right)} f_{i} \sim \frac{2^{N-1}}{\sqrt{3}} f_{i}
$$

although we have $\left|n_{i j}\right| \leq 1$.

\section{CONCLUSION}

Natural inflation provides an attractive framework for large field chaotic inflation which can explain the recent detection of primordial gravitational waves by BICEP2. The KNP alignment mechanism offers an interesting scheme to get a super-Planckian effective axion decay constant $f_{\text {eff }} \gg M_{P l}$, which is a necessary component of natural inflation, starting from sub-Planckian fundamental axion decay constants $f_{i} \ll M_{P l}$ of multiple axions. In this paper, we extended the original KNP model with two axions to models with $N>2$ axions. Compared to the original KNP model, a notable difference is that large anomaly coefficients of $\mathcal{O}\left(f_{\text {eff }} / f_{i}\right)$ are not required anymore if the number of axions is moderately large as $N \ln N \gtrsim 2 \ln \left(f_{\text {eff }} / f_{i}\right)$. With such $N$, the effective decay constant can be exponentially

enhanced as $f_{\text {eff }} / f_{i} \sim \sqrt{N !} n^{N-1}$ for $n$ denoting the typical size of the integer-valued anomaly coefficients, and the probability for the necessary alignment to be achieved under a random choice of the anomaly coefficients is of the order of $f_{i} / f_{\text {eff }}$. 
The structure of our setup is rich enough to realize a variety of different possibilities.

For instance, it can realize a multiple axion monodromy $\prod_{i=2}^{N} Z_{n_{i}}$ yielding $f_{\text {eff }} / f_{i} \sim \prod_{i=2}^{N} n_{i}$. The setup can also give rise to an exponentially enhanced effective axion decay constant as $f_{\text {eff }} / f_{i} \sim 2^{N-1}$, even when all the integer-valued anomaly coefficients are restricted as $\left|n_{i j}\right| \leq 1$.

\section{Acknowledgements}

We thank Chang Sub Shin, Kwang Sik Jeong and Jinn Ouk Gong for helpful discussions.

[1] P. A. R. Ade et al. [BICEP2 Collaboration], Phys. Rev. Lett. 112, 241101 (2014) arXiv:1403.3985 [astro-ph.CO]].

[2] D. H. Lyth, Phys. Rev. Lett. 78, 1861 (1997) hep-ph/9606387.

[3] K. Freese, J. A. Frieman and A. V. Olinto, Phys. Rev. Lett. 65, 3233 (1990); F. C. Adams, J. R. Bond, K. Freese, J. A. Frieman and A. V. Olinto, Phys. Rev. D 47, 426 (1993) hep-ph/9207245.

[4] K. Freese and W. H. Kinney, arXiv:1403.5277 [astro-ph.CO].

[5] K. Choi and J. E. Kim, Phys. Lett. B 154, 393 (1985) [Erratum-ibid. 156B, 452 (1985)];

P. Svrcek and E. Witten, JHEP 0606, 051 (2006) hep-th/0605206; T. Banks, M. Dine, P. J. Fox and E. Gorbatov, JCAP 0306, 001 (2003) hep-th/0303252.

[6] J. E. Kim, H. P. Nilles and M. Peloso, JCAP 0501, 005 (2005) hep-ph/0409138.

[7] N. Arkani-Hamed, H. -C. Cheng, P. Creminelli and L. Randall, Phys. Rev. Lett. 90, 221302 (2003) hep-th/0301218.

[8] C. Germani and A. Kehagias, Phys. Rev. Lett. 106, 161302 (2011) [arXiv:1012.0853 [hep-ph]]; C. Germani and Y. Watanabe, JCAP 1107, 031 (2011) [Addendum-ibid. 1107, A01 (2011)] arXiv:1106.0502 [astro-ph.CO]].

[9] S. Dimopoulos, S. Kachru, J. McGreevy and J. G. Wacker, JCAP 0808, 003 (2008) hep-th/0507205.

[10] A. R. Liddle, A. Mazumdar and F. E. Schunck, Phys. Rev. D 58, 061301 (1998) astro-ph/9804177]; E. J. Copeland, A. Mazumdar and N. J. Nunes, Phys. Rev. D 60, 
083506 (1999) astro-ph/9904309]; P. Kanti and K. A. Olive, Phys. Lett. B 464, 192 (1999) hep-ph/9906331.

[11] E. Silverstein and A. Westphal, Phys. Rev. D 78, 106003 (2008) arXiv:0803.3085 [hepth]]; L. McAllister, E. Silverstein and A. Westphal, Phys. Rev. D 82, 046003 (2010) arXiv:0808.0706 [hep-th]].

[12] F. Marchesano, G. Shiu and A. M. Uranga, arXiv:1404.3040 [hep-th]; A. Hebecker, S. C. Kraus and L. T. Witkowski, arXiv:1404.3711 [hep-th].

[13] N. Kaloper and L. Sorbo, Phys. Rev. Lett. 102, 121301 (2009) [arXiv:0811.1989 [hep-th]]; N. Kaloper, A. Lawrence and L. Sorbo, JCAP 1103, 023 (2011) arXiv:1101.0026 [hep-th]].

[14] K. Harigaya and M. Ibe, arXiv:1404.3511 [hep-ph].

[15] S. -H. H. Tye and S. S. C. Wong, arXiv:1404.6988 [astro-ph.CO]

[16] I. Ben-Dayan, F. G. Pedro and A. Westphal, arXiv:1404.7773 [hep-th].

[17] A. Chatzistavrakidis, E. Erfani, H. P. Nilles and I. Zavala, JCAP 1209, 006 (2012) arXiv:1207.1128 [hep-ph]]. 